Radioprotection, Suppl. 1, vol. 40 (2005) S839-S844

(C) EDP Sciences, 2005

DOI: 10.1051/radiopro:2005s1-123

\title{
ASTRAL V2.2: A new version to better assess post-accidental situations
}

\author{
Ph. Calmon ${ }^{1}$ and C. Mourlon ${ }^{2}$ \\ ${ }^{1}$ Institute of Radioprotection and Nuclear Safety (IRSN), CE/Cadarache, BP. 3, \\ 13115 St. Paul-lez-Durance Cedex, France \\ ${ }^{2}$ Division of Environment and Installations, Laboratory of Environmental Modelling
}

\begin{abstract}
Should an important release of radionuclides occur in the environment, one would quickly have to assess radionuclide concentration in media and foodstuff, deduce the potential exposition of concerned populations to the radiations, predict the evolution of the situation and propose different scenarios of managing the contaminated zones. To achieve this, the ASTRAL code was created, which can be used by a relatively large group of people composing crisis centres or making predictive impact studies. The starting point of the assessments is the ground deposit of radionuclides: the atmospheric dispersion phase and the cloud exposure are not taken into account here. The calculations achieved concern time evolution of radionuclide concentration in compartments of the agricultural and forest food chain as well as assessment of doses due to external and internal exposure. These values are compared to regulation limits and/or intervention levels. Different simulations of contaminated zones management can be led by applying counter-measures, dispositions meant to reduce the accident's impact on environment and populations. The seventy radionuclides taken into account in the present version are some of those met in case of accidents or during the exploitation of a Pressurized Water Reactor (PWR). They relate to 37 elements: Ag, Am, Ba, Br, Cd, Ce, Cl, Cm, Co, Cr, Cs, Eu, Fe, I, Ir, La, Mn, Mo, Nb, Nd, Ni, Np, Pm, Pr, Pu, Rb, $\mathrm{Rh}, \mathrm{Ru}, \mathrm{Sb}, \mathrm{Sm}, \mathrm{Sn}, \mathrm{Sr}, \mathrm{Tc}, \mathrm{Te}, \mathrm{Y}, \mathrm{Zn}, \mathrm{Zr}$. On a temporal point of view, the predictions extend to 3 years after the deposition. The internet technology chosen for this version offers important advantages: the spreading and maintenance of the tool are very easy, the code may be installed on a portable computer, the access to results is much quicker, the interface is very user friendly, so that no training is needed to perform calculations. As for any radioecological tool, a large number of parameters intervene in the calculations. All parameters can be modified, even though different user profiles grant different rights in handling them.
\end{abstract}

\section{INTRODUCTION}

Whether for studying impacts of a hypothetical nuclear accident or for handling a crisis situation, experts and decision-makers, after a large scale atmospheric radioactive release, want to assess the contamination of the environment and food products, derive from it the potential exposure of populations to ionising radiations, predict the evolution of the situation and propose different scenarios for the management of contaminated zones. Starting from estimations or measurements of a radionuclide deposit on the ground, the ASTRAL (acronym for "Technical Assistance for post-Accidental Radioprotection”) computer code meets these demands: it calculates the evolution of concentrations in the food chain components and the doses to man due to external exposure and internal intake through inhalation of resuspended radionuclides and ingestion of contaminated foodstuffs. Furthermore, various counter-measures can be simulated and therefore the efficiency of different managements of contaminated zones and products can be evaluated. The ASTRAL code was developed as part of a project cofunded by IRSN and EDF (Electricité de France, the French national electric facility). The present version of ASTRAL has been released in 2003, and is installed in IRSN's Crisis Technical Centre and in EDF. A demonstration of this version is proposed. 
The seventy radionuclides taken into account in the present version are some of those met in case of accidents or during the exploitation of a Pressurized Water Reactor (PWR). They relate to 37 elements: Ag, Am, Ba, Br, Cd, Ce, Cl, Cm, Co, Cr, Cs, Eu, Fe, I, Ir, La, Mn, Mo, Nb, Nd, Ni, Np, Pm, Pr, Rb, $\mathrm{Rh}, \mathrm{Ru}, \mathrm{Sb}, \mathrm{Sm}, \mathrm{Sn}, \mathrm{Sr}, \mathrm{Tc}, \mathrm{Te}, \mathrm{Y}, \mathrm{Zn}, \mathrm{Zr}$. On a temporal point of view, the predictions extend to 3 years after the deposition.

\section{CALCULATIONS PERFORMED}

The environment studied comprehends the agricultural and forest ecosystems. The calculations performed are radionuclide concentrations in different products of these two ecosystems and doses to man with different exposure scenarios and for different age groups (detailed information in IRSN report for ASTRAL V2 [1]).

For the agricultural ecosystem, different food types refer to different equation sets. For plants, three types are distinguished: annual crops, market garden produce and pasture grass. For animal products, contamination being attributed to ingestion, concentration is derived from that of the plants, using daily intakes defined by feeding calendars. For the forest ecosystem, three food types refer to different equation sets: mushrooms, berries and game. The radionuclide concentrations and their time evolution are then compared to maximum permitted levels (figure 1). The default values for the limits are those of the Euratom regulation, but can as well be any limit required by the decision maker.

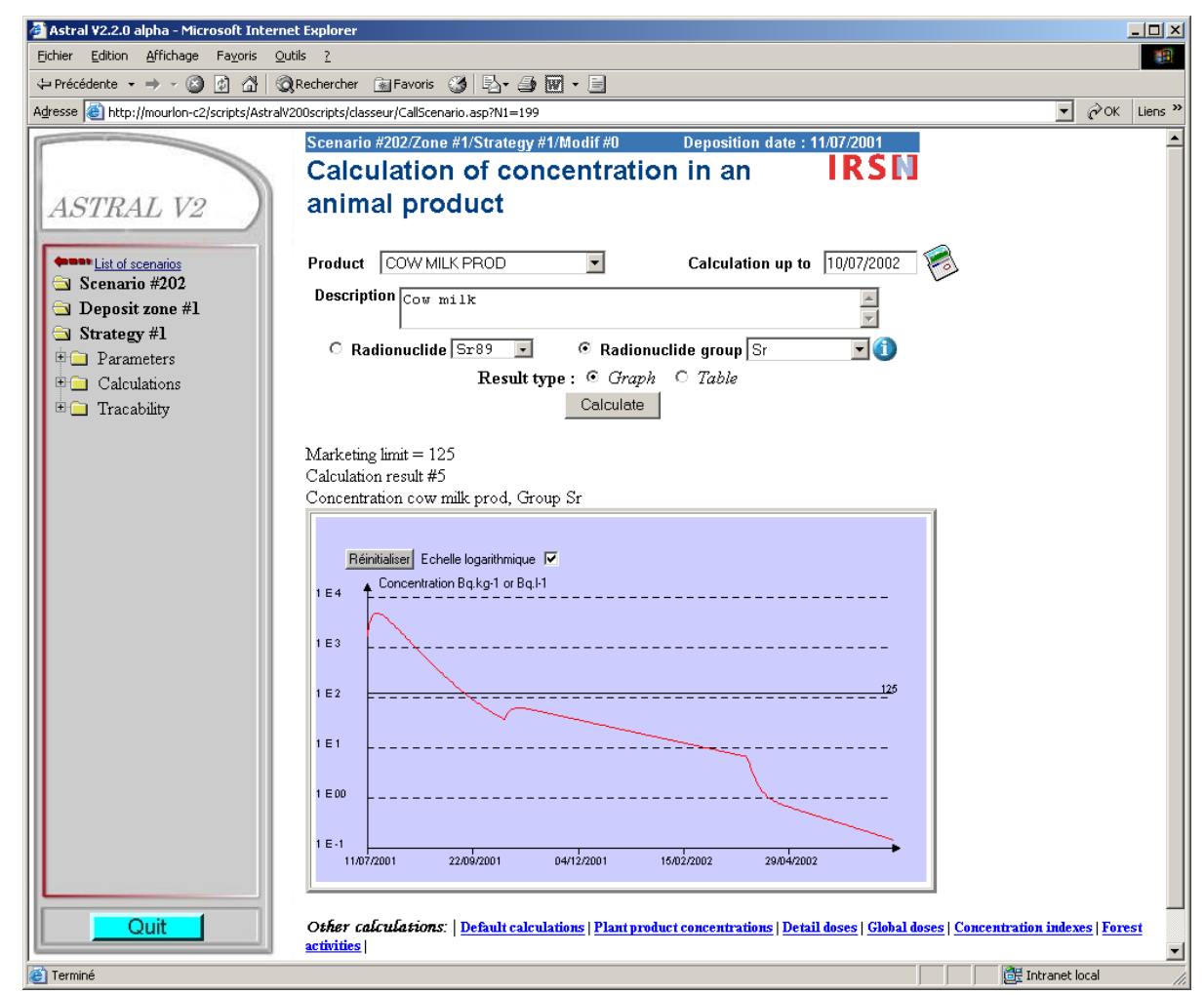

Figure 1. Strontium group concentration in cow milk versus time, compared to Euratom regulation limit.

The exposure pathways of man are external exposure to deposits, inhalation of resuspended particles, and ingestion of contaminated foodstuff. The accidental phase doses - due to cloud shine or inhalation in the plume - are not considered. The doses calculated in ASTRAL day by day or on a user defined time period can be effective doses or thyroid doses, in the case of contamination (inhalation and ingestion). 
Nine exposure scenarios are proposed for dose calculations, reflecting six age groups - as defined in the ICRP 72 [2]- and different lifestyles such as rural dwellers, hunters, berry and mushroom gatherers and forest workers. Most parameters, such as the diet, contaminated fraction of foodstuff or time spent in different environments, can be modified by the user, enabling him to create his own exposure scenario. The results are presented as tables and pie-charts, making easier the sighting of critical exposure pathways, radionuclides and foodstuffs (see figure 2).

While in a first stage calculations are performed to evaluate the evolution of the situation with no human intervention, it is possible that in future stages, one will want to see how the initial situation evolves with different ways of handling it. Diverse counter-measures can be simulated in ASTRAL, some aimed at reducing concentrations of foodstuff, others at limiting exposition of man:

- $\quad$ for agricultural plants: use of fertilizers and/or lime, deep ploughing, top soil layer stripping;

- $\quad$ for agricultural animal products: use of Prussian blue or clay, change in feeding - especially interruption of grazing;

- $\quad$ for man: change in the food intake, ban of consumption of local products, modification of time budgets in different environments...

By comparing the levels reached by concentrations and doses in these various cases, the efficiency of different managements of contaminated zones can thus be evaluated.

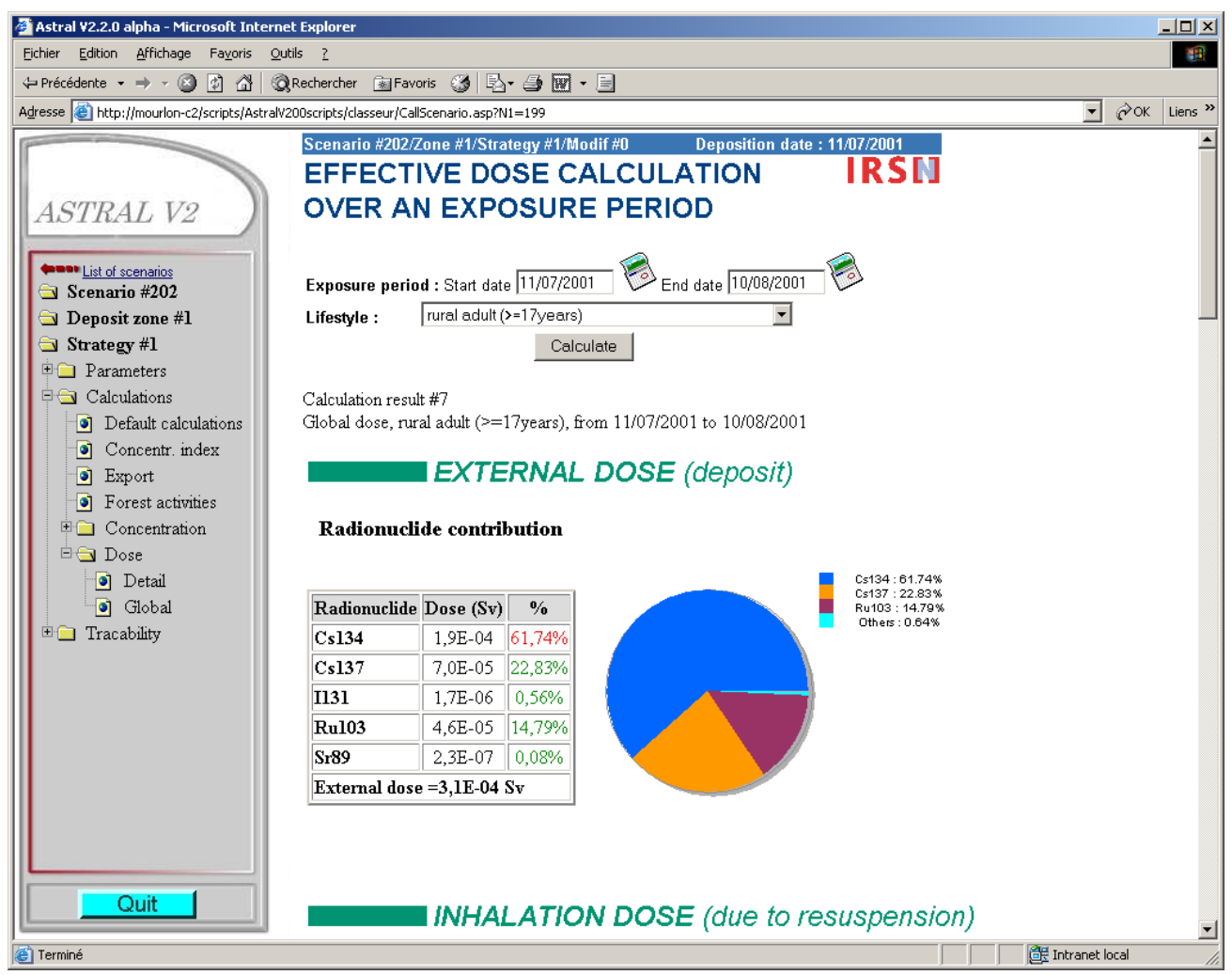

Figure 2. Example of dose presentation for a given time period and individual type.

\section{CHARACTERISTICS OF THE PRESENT VERSION}

The development of the version "V2.x" started in 1999 and has been subject to a quality assurance program. The identified customers and users have been associated in a control committee expressing needs, validating choices and checking the good progress of the project. 


\subsection{Technology and architecture}

The ASTRAL software uses an internet technology and has an architecture of a three-third type, requiring an Oracle database, a Microsoft web server and a consultation station. These choices offer important advantages:

- the spreading and maintenance of the tool are very easy. In fact, once the server and the database have been installed on a network, the users only need a web browser to run the code. The initial installation and the updating are thus made only on the server and/or the database.

- the code may be installed on a portable computer, which hosts the database, the internet server and the web browser. This enables working isolated from the network, on the field or in decision making sites.

- the access to results is very quick, for they are calculated or saved only on decision of the user.

- The very user friendly interface, so that no training is needed to perform calculations.

\subsection{Ergonomics}

As for any radioecological tool, a large number of parameters intervene in the calculations. All parameters can be modified, even though different user profiles grant different rights in handling them. Also, to define his request, the user must choose many input data (radionuclide, foodstuff, lifestyle, dates...). As a result of this, radioecological tools can easily end up with abundant and complicated screens. To avoid this problem, special care was given to ergonomics.

\subsubsection{Making use easier}

One of the goals in developing the tool was to make it clear enough to enable people to use it with as little training as possible, if any. Several devices were used to achieve this, such as:

- list boxes for selecting entities, tick boxes, calendars icons

- $\quad$ hypertext links to get from one place to another (see bottom of figure 1).

- $\quad$ help buttons 1 in the screens.

\subsubsection{Making result interpretation and comprehension easier}

Whenever possible, the default presentation of results is a graph, but the possibility is always given to shift to a table presentation. Graphs are meant to be synthetic; however precise information must remain reachable. Therefore small applications have been developed: the user can zoom in on parts of the graphs, getting the detailed information of values and dates in a dedicated information box. He may also shift from linear to logarithmic scales by using a click box (see figure 1).

ASTRAL can be used in crisis situations, in which case its results are intended to be communicated to decision makers, usually people not belonging to the scientific world. The information thus cannot just consist of values such as $\mathrm{Bq} / \mathrm{kg}$ concentrations for foodstuff: results must be understandable and interpretable by non-specialists and must always be compared to some limit. An example of the efforts deployed to make information clear and communicable is the ratio of the concentration to the limit: it is allocated to a group depending on whether it is smaller than $30 \%$ (green colour assigned), between $30 \%$ and $300 \%$ (orange) or bigger than $300 \%$ (red). This processing of the information, with the use of colours for each group, enables a quick evaluation of the products and time periods where problems will be encountered and may help to prioritise decisions and counter-measures (see figure 3). 


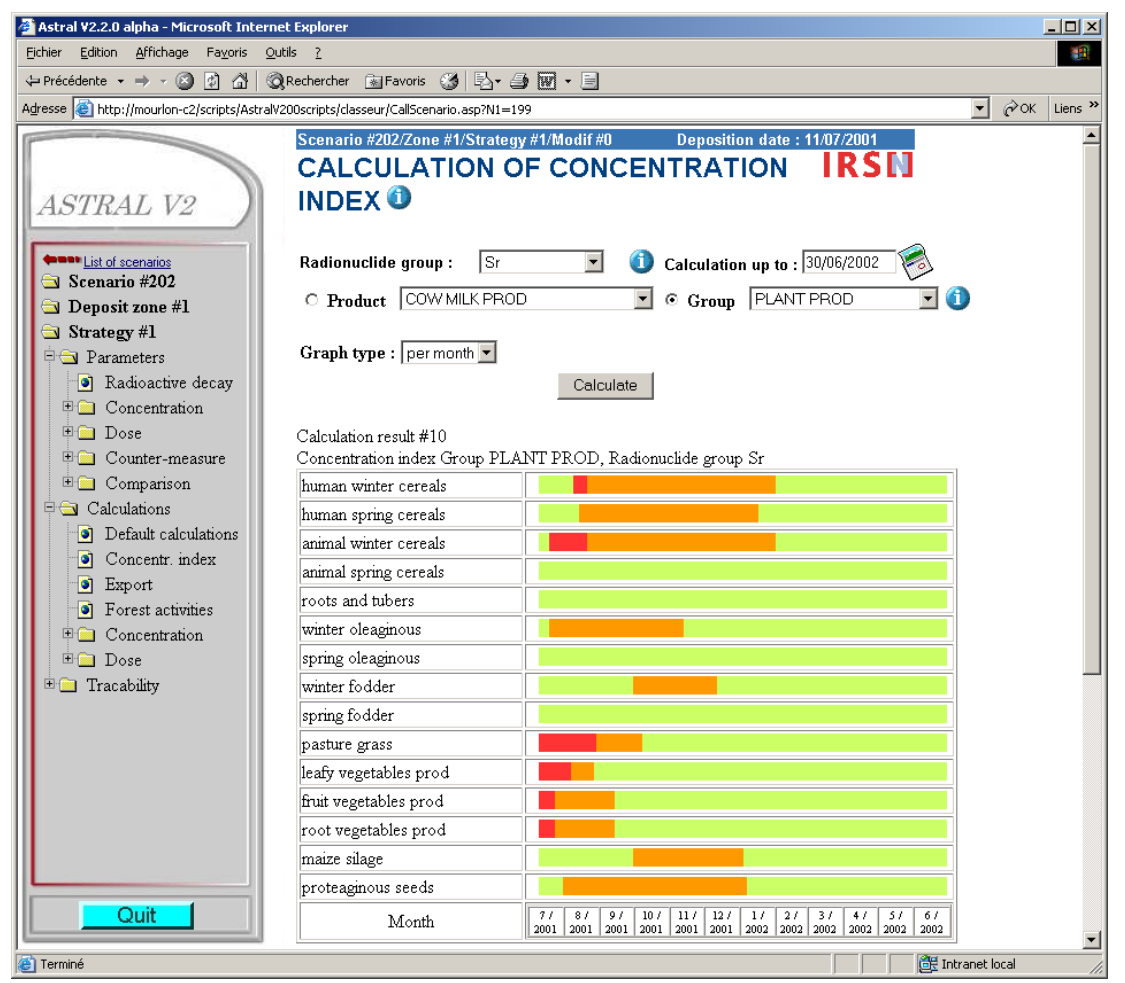

Figure 3. Time evolution of the concentration/limit ratio, displayed with three colours depending on its value: enabling a quick identification of problematic products and time periods.

\subsubsection{Default results}

The tool enables the calculation of a wide variety of results. However, the feedback from IRSN's participation in crisis exercises has shown that, too dense information doesn't help decision makers and that the latter only want a few results - often the same. Therefore the endpoints that experience has taught to be most relevant or systematically asked for have been grouped in a specific result screen. This "default results" screen launches the following calculations:

- concentration in milk after the first day and at the day the maximum is reached, for each radionuclide group, using the three colour convention for comparison to the limit;

- concentration in leafy vegetables after the first day and after the first week, for each radionuclide group, using the three colour convention for comparison to the limit;

- doses after one day, one week, one month and one year for six individual types: baby ( $0-1$ year), very young children (1-2 y), rural young children (2-7 y), rural children (7-12 y), rural teenagers (12-17 y) and rural adults (above $17 \mathrm{y}$ ). For each of these time periods and individual types, the effective doses are calculated - for all radionuclides, all exposure pathways.

These results, to be used in a first phase of a crisis, present the state of the usually most sensible foodstuff (milk and leafy vegetables) and enable the identification of the most vulnerable individual types - as well as the related critical time periods. 


\subsection{Links with other tools}

The starting point of calculations, deposits on the ground, can be issued from field measurements or predicted by a code - especially in an early phase when deposits are expected but have not occurred. Such predictions are performed by CONRAD, the atmospheric dispersion tool developed by IRSN for its Crisis Technical Centre. A link in ASTRAL has been developed to fetch CONRAD's results and input them in its database.

The results proposed by ASTRAL can in turn be saved in its database, on the user's request, in order to be processed by other tools such as Geographical Information Systems, which enable crossing spatial information and producing maps. Such maps are very helpful in crisis situations to decision makers since by answering to questions of a "where?" type, at a given time, they complete the information delivered by ASTRAL, which answers to questions of a "when?" type, at a given site. This is why a link to ASTRAL is under construction in CARTINFO, an IRSN tool dedicated to mapping results for its Crisis Technical Centre.

Thus the CONRAD, ASTRAL and CARTINFO softwares constitute a chain for IRSN's Crisis Technical Centre, calculating transfers through the environment and consequences of an atmospheric accidental radionuclide release.

\subsection{Latest achievements}

In 2003, a securized extranet access has been developed, that allows an authorized connection outside IRSN. The web address is: https://www.irsn-astral.org. The use of ASTRAL via extranet is conditioned by a login and a password, that could be delivered on request.

Since the extranet version of ASTRAL was developed, the entire translation of the software and the linked documentation into English was also realised in 2004. The English version of ASTRAL can be accessed via the selection of the English flag on the welcome page of the software.

\section{CONCLUSION}

The ASTRAL software has been developed to assess the contamination of the agricultural and forest environments and food products, calculate the potential exposure of populations to ionising radiations, predict the evolution of the situation and propose different scenarios for the management of contaminated zones after an atmospheric radioactive release. ASTRAL could be used to handle a crisis situation or in the frame of impact studies. The software offers the possibility to make calculations with 70 radionuclides. Web technology and ergonomics allow an easy installation, spreading and maintenance of the tool, a user friendly interface, a quick access to results and a permanent tracability between scenario data and results. ASTRAL can be accessed either by intranet or now also by extranet in two languages: French and English. At the end of this year, a new version will propose to make calculations with some of the most important isotopes of uranium.

\section{References}

[1] Calmon P. and Mourlon C., ASTRAL V2.1 code - Equations and Parameters, (2003), report IRSN/DPRE/SERLAB $n^{\circ} 03-16$.

[2] International Commission on Radiological Protection. Age-dependent Dose to Members of the Public from Intake of Radionuclides: Part 5 Compilation of Ingestion and Inhalation Dose Coefficients, Publication 72, (Pergamon Press, Oxford, 1996). 